\title{
FOUR-DIMENSIONAL PATH INTEGRATION METHOD FOR ESTIMATING THE STOCHASTIC ROLL RESPONSE
}

\author{
Wei Chai ${ }^{1}$, Arvid Naess ${ }^{2,3}$, and Bernt J. Leira ${ }^{1}$ \\ ${ }^{1}$ Department of Marine Technology, Norwegian University of Science and Technology \\ Otto Nielsens veg 10, Department of Marine Technology, NTNU, 7491 Trondheim, Norway \\ \{chai.wei, bernt.leira\}@ntnu.no \\ ${ }^{2}$ Centre for Ships and Ocean Structures, Norwegian University of Science and Technology \\ Otto Nielsens veg 10, Department of Marine Technology, NTNU, 7491 Trondheim, Norway \\ \{arvid.naess\}@math.ntnu.no \\ ${ }^{3}$ Department of Mathematical Sciences, Norwegian University of Science and Technology \\ Sentralbygg 2, 11th floor, room 1144, Gløshaugen, 7491 Trondheim, Norway \\ \{arvid.naess\}@math.ntnu.no
}

Keywords: Path integration method, nonlinear ship rolling, stochastic response.

\begin{abstract}
In this paper, the Markov theory is introduced in order to model the roll motion excited by random wave excitation. Specifically, a four-dimensional (4D) Markov dynamic system is established by combing the single-degree-of-freedom (SDOF) model used to represent the ship rolling behavior in random beam seas with a second-order linear filter used to approximate the stationary wave excitation moment. Based on the Markov property of the 4D coupled dynamic system, a 4D path integration (PI) method is applied in order to solve the Fokker-Planck equation, which governs the probabilistic properties of the coupled dynamic system. The theoretical principle and numerical implementation of the current state of the art 4D PI method are presented. Furthermore, the numerical robustness and accuracy of the 4D PI method are evaluated by means of the versatile Monte Carlo simulation (MCS) technique.
\end{abstract}




\section{INTRODUCTION}

The challenge to calculate the response statistics of nonlinear, marine structures subjected to a random seaway is still substantial. In this work, the rolling behavior of a vessel in random beam seas is to be studied since the associated high-level responses with low probability levels are important for reliability based ship design and operation in practice.

For the cases of beam seas, the roll motion is assumed to be decoupled from other motions and governed by a single-degree-of-freedom (SDOF) equation [1], which is also a second order differential equation. In the SDOF model, the nonlinearities associated with damping and restoring terms as well as the randomness of the wave excitation term are all incorporated.

Various probabilistic approaches have been proposed to analyze the stochastic response of the roll motion in random seas [2-4]. Among these strategies, the methodology based on the Markov diffusion theory is attractive because the probabilistic properties of the roll motion is governed by the Fokker-Planck (FP) equation. In order to build a Markov system for describing the random roll motion, a second order linear filter is applied in order to approximate the stationary wave excitation moment as a filtered white noise. Subsequently, a four-dimensional (4D) coupled system is generated but the corresponding high-dimensional FP equation leads to another challenge.

Analytical solutions of the FP equations are only available for some linear systems and a very limited class if nonlinear systems. Direct numerical methods aiming to solve the lowdimensional FP equations, such as the finite element method [5] and the finite difference method [6], are hardly feasible because for the high-dimensional FP equations. In this regard, the so-called "curse of dimension" problem comes into play, which means that difficulties arise due to the processing capacity as well as the storage needed for the computation increases dramatically with the dimension of the FP equation.

The path integration (PI) method is an efficient approximation for solving the FP equation and providing the stationary or non-stationary response probability density function (PDF) of the dynamic system [7-9]. The evolution of the response PDF is computed in short time steps via a step-by-step solution technique. Specifically, the based on the Chapman-Kolmogorov equation, the response PDF at a given time instant can be obtained when the response PDF at an earlier close time as well as the conditional PDF are already known. In this work, a 4D PI procedure is developed to address the challenge of determining the response statistics of the roll motion in random beam seas. The feasibility and reliability of the PI technique will be demonstrated by comparing with the Monte Carlo simulation.

\section{MATHEMATICAL MODEL}

When the ship is excited by beam wave loads, the roll motion is described by the following SDOF equation [10]:

$$
\ddot{\theta}(t)+b_{44} \dot{\theta}(t)+b_{44 q} \dot{\theta}(t)|\dot{\theta}(t)|+c_{1} \theta(t)-c_{3} \theta^{3}(t)=m(t)
$$

where $\theta(t)$ and $\dot{\theta}(t)$ are the roll angle and the roll velocity, respectively. $b_{44}$ and $b_{44 q}$ are the relative linear and quadratic damping coefficients, $c_{1}$ and $c_{3}$ are the relative linear and nonlinear roll restoring coefficients. $m(\mathrm{t})$ is the relative roll excitation moment, it is a stationary Gaussian process and described by the spectrum $S_{m m}(\omega)$. The SDOF model (1) can be transformed into the following state-space equation which is written as:

$$
\left\{\begin{array}{l}
d x_{1}=x_{2} d t \\
d x_{2}=\left(-b_{44} x_{2}-b_{44 q} x_{2}\left|x_{2}\right|-c_{1} x_{1}+c_{3} x_{1}^{3}+x_{3}\right) d t
\end{array}\right.
$$


where $x_{1}=\theta(t), x_{2}=\dot{\theta}(t)$ and $x_{3}=m(t)$ is the driving process for the dynamic system (2).

The linear filter technique is widely used in the engineering community due to its simplicity and practicality. The driving process $m(t)$ is a stationary Gaussian process with appropriate spectral density, it can approximated quite satisfactorily by a suitable linear filter. In this work, a second-order linear is applied in order to approximate the desired spectrum, $S_{m m}(\omega)$.

The second-order linear filter is expressed by the following differential equation:

$$
\left\{\begin{array}{l}
d x_{3}=\left(x_{4}-\beta x_{3}\right) d t+\gamma d W \\
d x_{4}=-\alpha x_{3} d t
\end{array}\right.
$$

where $x_{3}$ and $x_{4}$ are the state variables in the filter equation with $x_{3}$ representing the output $m(t) . d W(t)=W(t+d t)-W(t)$ represents an infinitesimal increment of a standard Wiener process with $\mathrm{E}\{d W(t)\}=0$ and $\mathrm{E}\{d W(t) d W(s)\}=0$ for $t \neq s$ and $\mathrm{E}\left\{d W(t)^{2}\right\}=0$. The spectrum generated by the second-order linear filter (3) is denoted as $S_{\text {Filter }}(\omega)$ and given as:

$$
S_{\text {Filter }}(\omega)=\frac{1}{2 \pi} \frac{\gamma^{2} \omega^{2}}{\left(\alpha-\omega^{2}\right)^{2}+(\beta \omega)^{2}}
$$

where $\alpha, \beta, \gamma$ are the parameters of the linear filter and they are determined by minimizing the least square error between the spectral density of the filtered spectrum $S_{F i l t e r}(\omega)$ and the spectral density of the target spectrum, $S_{m m}(\omega)$. It is worth emphasizing that the filtered spectrum (4) is double-sided, while the relative roll excitation spectrum $S_{m m}(\omega)$ is physically singlesided. This difference needs to be reflected when performing the simulation itself.

By combining the equation (2) with equation (3), the extended dynamic system is formed. Therefore, the roll motion in random beam seas can be described by the following 4D state space equation:

$$
\left\{\begin{array}{l}
d x_{1}=x_{2} d t \\
d x_{2}=\left(-b_{44} x_{2}-b_{44 q} x_{2}\left|x_{2}\right|-c_{1} x_{1}+c_{3} x_{1}^{3}+x_{3}\right) d t \\
d x_{3}=\left(x_{4}-\beta x_{3}\right) d t+\gamma d W \\
d x_{4}=-\alpha x_{3} d t
\end{array}\right.
$$

\section{4D PATH INTEGRATION METHOD}

\subsection{Main principle}

The dynamic system represented by equation (5) is a Markov diffusion process and it can be expressed as the following stochastic differential equation:

$$
d \mathbf{x}=\mathbf{a}(\mathbf{x}, t) d t+\mathbf{b}(t) d \mathbf{W}(t)
$$

where $\mathbf{x}(t)=\left(x_{1}(t), \ldots, x_{4}(t)\right)^{\mathrm{T}}$ is a $4 \mathrm{D}$ state space vector process, the vector $\mathbf{a}(\mathbf{x}, t)$ is the drift term and $\mathbf{b}(t) d \mathbf{W}(t)$ represents the diffusive term. The vector $d \mathbf{W}(t)=\mathbf{W}(t+d t)-\mathbf{W}(t)$ denotes independent increments of a standard Wiener process.

For the Markov process $\mathbf{x}(t)$, its transition probability density, $p\left(\mathbf{x}, t \mid \mathbf{x}^{\prime}, t^{\prime}\right)$, also known as the conditional PDF is governed by the FP equation, which is expressed as:

$$
\frac{\partial}{\partial t} p\left(\mathbf{x}, t \mid \mathbf{x}^{\prime}, t^{\prime}\right)=-\sum_{i=1}^{4} \frac{\partial}{\partial x_{i}} a_{i}(\mathbf{x}, t) p\left(\mathbf{x}, t \mid \mathbf{x}^{\prime}, t^{\prime}\right)+\frac{1}{2} \sum_{i=1}^{4} \sum_{j=1}^{4} \frac{\partial^{2}}{\partial x_{i} \partial x_{j}}\left(b(t) \cdot b^{T}(t)\right)_{i j} p\left(\mathbf{x}, t \mid \mathbf{x}^{\prime}, t^{\prime}\right)
$$

where $\mathbf{x}^{\prime}$ denotes the state space vector at time $t^{\prime}$ and $t^{\prime}<t$. 
For numerical solution of the time continuous stochastic differential equation (SDE) (6), discretization of the equation with respect to time $t$ is essential. In this regard, Naess and Moe propose a fourth-order Runge-Kutta-Maruyama approximation:

$$
\mathbf{x}(t)=\mathbf{x}\left(t^{\prime}\right)+\mathbf{r}\left(\mathbf{x}\left(t^{\prime}\right), t^{\prime}\right) \Delta t+b\left(t^{\prime}\right) \Delta \mathbf{W}\left(t^{\prime}\right)
$$

where $\Delta t=t-t^{\prime}$ is the time increment and the vector $\mathbf{r}\left(\mathbf{x}\left(t^{\prime}\right), t^{\prime}\right)$ denotes the explicit fourth-order Runge-Kutta (RK4) increment or RK4 approximation. Since $\mathbf{W}(t)$ is a Wiener process, for short time increment $\Delta t$, the independent increment $\Delta \mathbf{W}\left(t^{\prime}\right)=\mathbf{W}(t)-\mathbf{W}\left(t^{\prime}\right)$ is a Gaussian variable for every $t^{\prime}$ (a constant is considered to be a Gaussian variable with zero standard deviation).

With the introduction of the time discrete approximation, the time sequence $\{\mathbf{x}(i \cdot \Delta t)\}_{i=0}^{\infty}$ is a Markov chain and it can approximate the time-continuous Markov solution of the SDE (6) with satisfactory accuracy when the time increment $\Delta t$ is sufficiently small [8].

For a time increment $\Delta t=t-t^{\prime}$, the transition probability density of equation (8), $p\left(\mathbf{x}, t \mid \mathbf{x}^{\prime}, t^{\prime}\right)$, is given as a (degenerate) Gaussian distribution, which is written as:

$$
\begin{aligned}
p\left(\mathbf{x}, t \mid \mathbf{x}^{\prime}, t^{\prime}\right)= & \delta\left(x_{1}-x_{1}^{\prime}-r_{1}\left(\mathbf{x}^{\prime}, t^{\prime}, \Delta t\right)\right) \cdot \delta\left(x_{2}-x_{2}^{\prime}-r_{2}\left(\mathbf{x}^{\prime}, t^{\prime}, \Delta t\right)\right) \\
& \cdot \tilde{p}\left(x_{3}, t \mid x_{3}^{\prime}, t^{\prime}\right) \cdot \delta\left(x_{4}-x_{4}^{\prime}-r_{4}\left(\mathbf{x}^{\prime}, t^{\prime}, \Delta t\right)\right)
\end{aligned}
$$

where $\tilde{p}\left(x_{3}, t \mid x_{3}^{\prime}, t^{\prime}\right)$ is given by the relation:

$$
\tilde{p}\left(x_{3}, t \mid x_{3}^{\prime}, t^{\prime}\right)=\frac{1}{\sqrt{2 \pi \gamma^{2} \Delta t}} \cdot \exp \left\{-\frac{\left(x_{3}-x_{3}^{\prime}-r_{3}\left(\mathbf{x}^{\prime}, t^{\prime}, \Delta t\right)\right)^{2}}{2 \gamma^{2} \Delta t}\right\}
$$

in which $r_{i}\left(\mathbf{x}^{\prime}, t^{\prime}, \Delta t\right)=r_{i}\left(\mathbf{x}\left(t^{\prime}\right), t^{\prime}, \Delta t\right), i=1,2,3,4$ are the Runge-Kutta increments for the state space variables.

For the PI method, the evolution of the response statistics, such as the PDF of the random process $\mathbf{x}$ at time $t$ can be obtained by the following basic equation:

$$
p(\mathbf{x}, t)=\int_{R^{4}} p\left(\mathbf{x}, t \mid \mathbf{x}^{\prime}, t^{\prime}\right) p\left(\mathbf{x}^{\prime}, t^{\prime}\right) d \mathbf{x}^{\prime}
$$

where $d \mathbf{x}^{\prime}=\prod_{i=1}^{4} d x_{i}^{\prime}$.

Since the expression for the transition probability density is obtained (i.e., the equations (9) and (10)), the PDF of $\mathbf{x}(t)$ can be obtained by the following iterative algorithm if an initial PDF (i.e. at time $t_{0}$ ) is given:

$$
p(\mathbf{x}, t)=\int_{R^{4}} \cdots \int_{R^{4}} \prod_{s=1}^{n} p\left(\mathbf{x}^{(s)}, t_{s} \mid \mathbf{x}^{(s-1)}, t_{s-1}\right) \cdot p\left(\mathbf{x}^{(0)}, t_{0}\right) d \mathbf{x}^{(0)} \ldots d \mathbf{x}^{(n-1)}
$$

where $\mathbf{x}=\mathbf{x}^{(n)}=\mathbf{x}\left(t_{n}\right), t=t_{n}=t_{0}+n \cdot \Delta t, \mathbf{x}^{(\mathrm{s})}=\mathbf{x}\left(t_{s}\right)$ and $t_{s}=t_{0}+s \cdot \Delta t$.

\subsection{Numerical implementation}

Equation (12) describes the mathematical principle of the PI approach. For numerical implementation of the 4D PI method, a reasonable computational domain and the corresponding computational grid have to be determined at first. In this work, the initial PDF $p\left(\mathbf{x}^{(0)}, t_{0}\right)$ is chosen as a 4D Gaussian PDF in four dimensions with zero mean and variances evaluated by a simple Monte Carlo simulation [8]. The straightforward Monte Carlo simulation (MCS) ensures that the initial 4D Gaussian PDF includes all the information corresponding to the selected parameters of the dynamic system, and it also provides a rational computational domain and computational grid for the subsequent simulation. 
As for the numerical implementation of the iterative algorithm (12) for each grid point, it represents the PDF at the previous time $t^{\prime}$ as an interpolating spline surface via Parabolic Bspline. Then, the RK4 approximation scheme is applied to calculate the deterministic trajectories backwards for each grid point. Therefore, each grid point at time $t$ is mapped backwards with the corresponding starting point at the previous time $t^{\prime}$ and the PDF values at the backward-mapped points, i.e. the starting points, can be given by using the spline surface. Finally, the PDF at time $t$, i.e. the new PDF, is calculated by substituting the transition probability density given in terms of equations (9) and (10) into the integral equation (11). The flowchart of the numerical implementation of the 4D PI method with the iterative algorithm described above is shown in Figure 1.

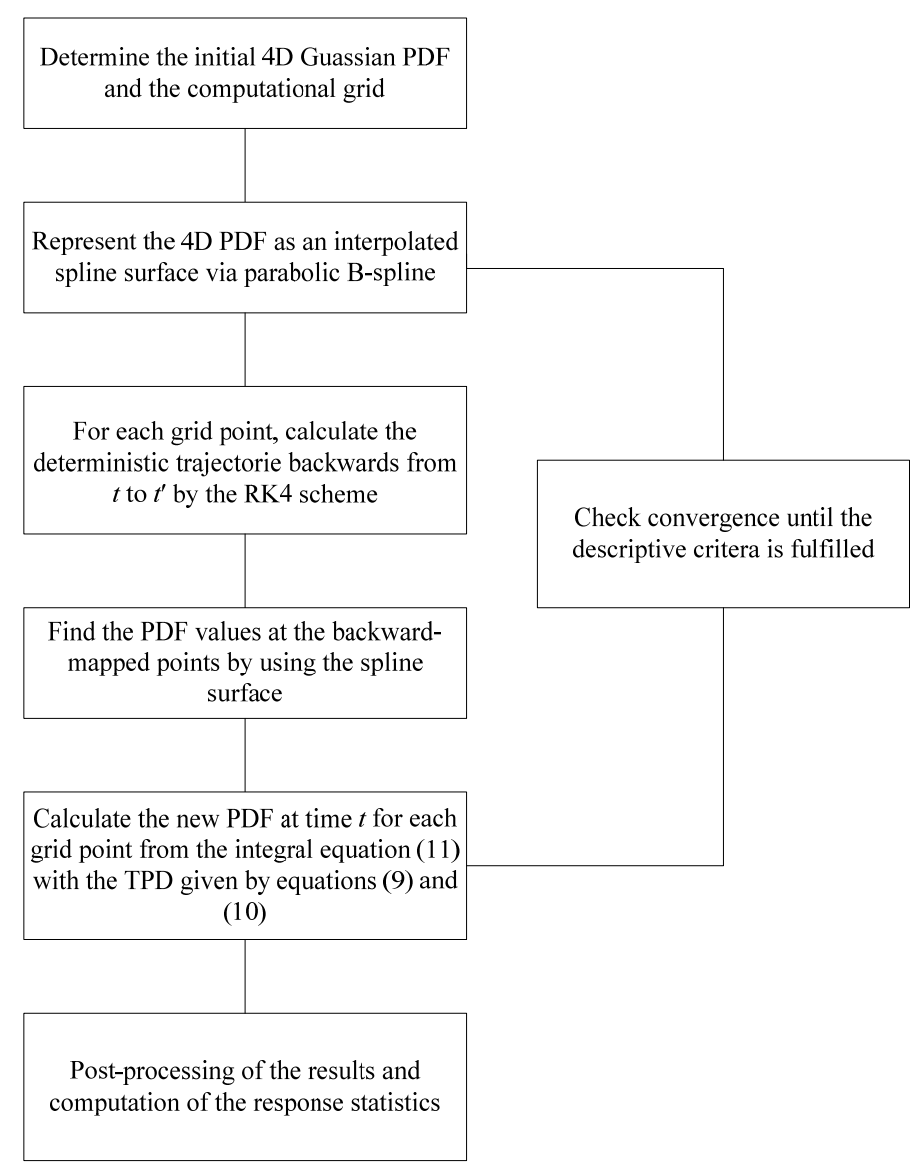

Figure 1: Flowchart of the numerical implementation of the 4D PI method

\section{NUMERICAL RESULTS}

In this Section, an ocean surveillance ship is selected in order to study the stochastic roll response. The parameters for this parameters are given in Ref. [11]. The relative roll excitation moment spectrum $S_{m m}(\omega)$ for a given sea state [10] is presented in Figure 2.

As mentioned above, the parameters $\alpha, \beta, \gamma$ in the second-order filter (4) are determined by minimizing the square errors between the filtered spectrum and the target spectrum, $S_{m m}$. It can be readily seen that the filtered spectrum is reasonable in terms of bandwidth, peak frequency and peak value. However, there is a slight discrepancy between the two spectra in the critical region near the natural roll frequency $\omega_{0}$. This discrepancy should not be neglected since the distribution of high level response is sensitive to the variation of the external excitation in this frequency region [12]. Therefore, a constant, $c$, should be introduced as a correc- 
tion factor for the filtered spectrum in order to decrease the discrepancy in the critical frequency region. The filtered spectrum (4) can then be changed into:

$$
S_{\text {Filter }}(\omega)=\frac{1}{2 \pi} \frac{(c \cdot \gamma)^{2} \omega^{2}}{\left(\alpha-\omega^{2}\right)^{2}+(\beta \omega)^{2}}
$$

and the corrected (or modified) spectrum in the critical region is also presented in Figure 2.

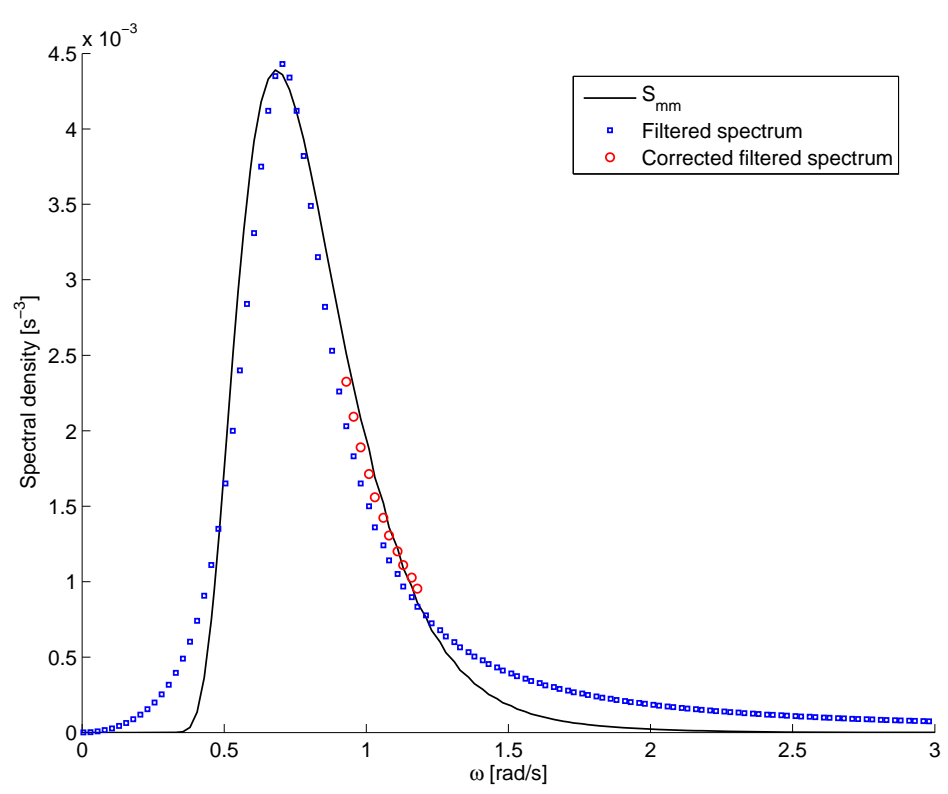

Figure 2: Relative roll excitation moment spectrum and the filtered spectrum, the corrected filtered spectrum (part) for a given sea state.

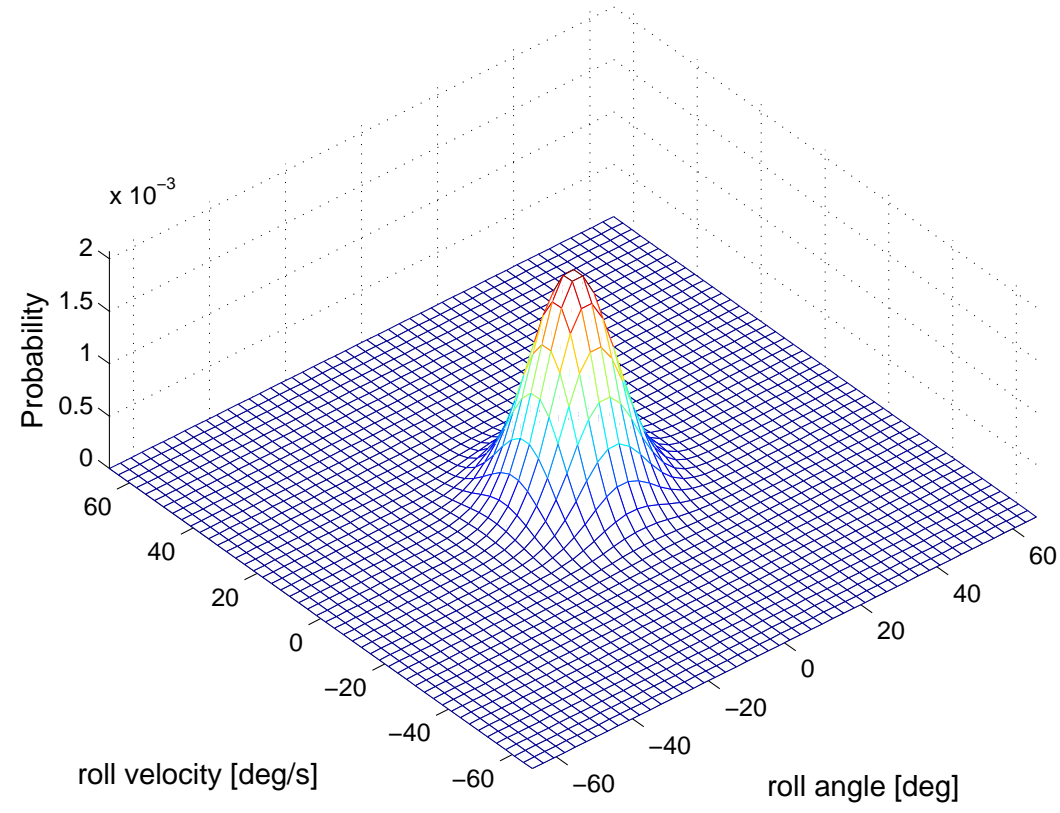

Figure 3: Joint PDF of the roll response obtained by the 4D PI method.

As mentioned in Section 3, the joint PDF of the roll angle process and the roll velocity process can be obtained directly by the 4D PI method. The joint PDF of the roll response yielded by the 4D PI method for the selected sea state is presented in Figure 3 and the mar- 
ginal PDFs of the roll angle process and the roll velocity process are shown in Figure 4 and Figure 5, respectively.

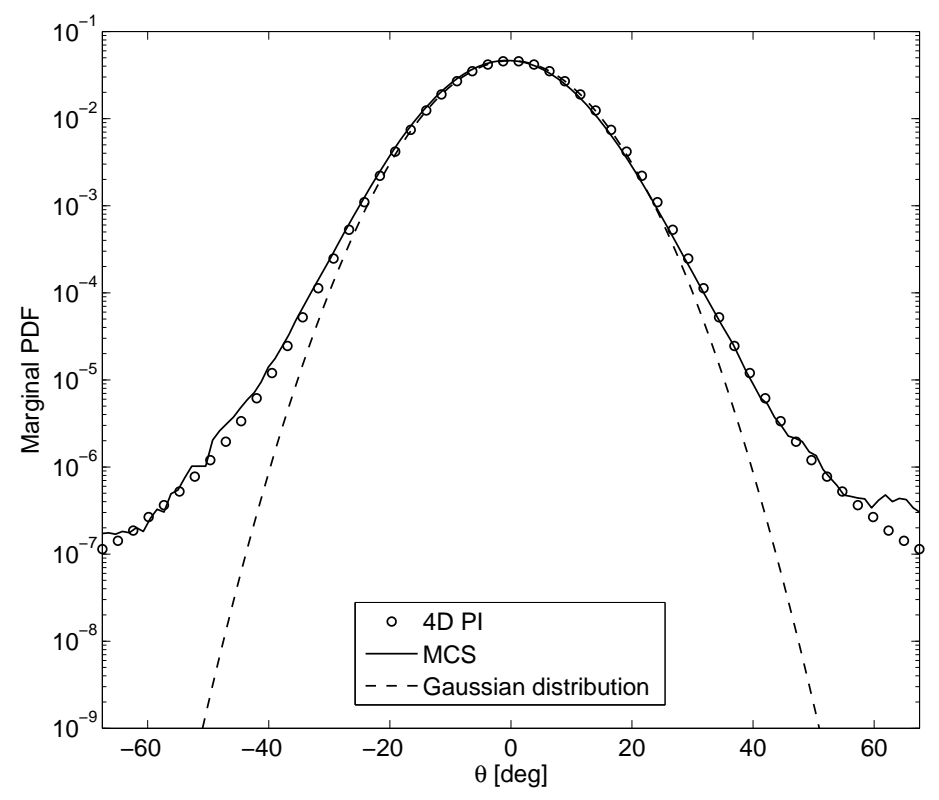

Figure 4: Marginal PDF of the roll angle process.

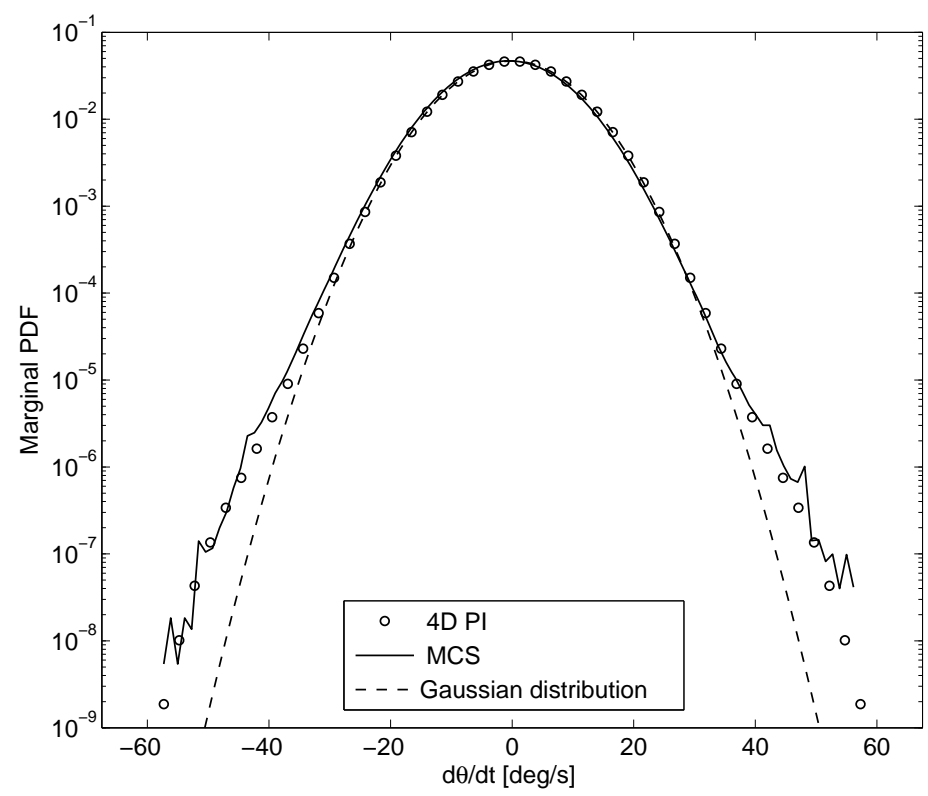

Figure 5: Marginal PDF of the roll velocity process.

In Figures 4 and 5, it is observed that the comparisons of the marginal PDFs obtained by the 4D PI method and the corresponding empirical estimations obtained by MCS demonstrate the high-level accuracy of the 4D PI method. Furthermore, the 4D PI method provides nice results for the low probabilities, but the distribution obtained by MCS are suffering from uncertainties. The Gaussian distributions of the marginal PDFs in Figures 4 and 5 are obtained by using the variances evaluated by the straightforward MCS technique mentioned in Section 3. Actually, they are the marginal PDFs of the 4D Gaussian PDF, $p\left(\mathbf{x}^{(0)}, t_{0}\right)$, which serves as the initial PDF in the 4D PI procedure. It is shown that the Gaussian distributions in Figures 4 and 5 provide reasonable approximations of the statistics of small amplitude roll motions. 
However, for the high-level responses, the distributions of the roll angle process and the roll velocity process are very different from the normal distribution, which underestimates the corresponding low levels in this region.

The mean upcrossing rate is a key parameter for estimation of the large and extreme response statistics as well as for evaluation of the associated reliability of marine structures subjected to random external excitations. The calculation of the mean upcrossing rate of the roll angle process $\theta(t)$ is usually based on the Rice formula (14) and the joint PDF of the roll response, which can be obtained directly by the 4D PI technique. The mean upcrossing rate $v^{+}(\zeta)$ is given as follows:

$$
\begin{aligned}
& v^{+}(\zeta)=\lim _{t \rightarrow \infty} v^{+}(\zeta ; t) \\
& v^{+}(\zeta ; t)=\int_{0}^{\infty} \dot{\theta} f_{\theta \dot{\theta}}(\zeta, \dot{\theta} ; t) d \dot{\theta}
\end{aligned}
$$

where $v^{+}(\zeta ; t)$ denotes the expected number of upcrossings for the $\zeta$-level per unit time at time $t$ by the roll angle process $\theta(t), f_{\theta \dot{\theta}}(\theta, \dot{\theta} ; t)$ is the joint PDF of the roll angle process and the roll velocity process at the time instant $t$.

The upcrossing rate for the selected sea state calculated by application of the 4D PI approach and the corresponding empirical estimation of the upcrossing rate as well as the 95\% confidence interval (CI) obtained by MCS are shown in Figure 6. It can be readily seen that the 4D PI technique yields quite accurate and reliable result, even in the high roll response region. Hence, the 4D PI method is suitable for evaluation of the response statistics with low probability levels.

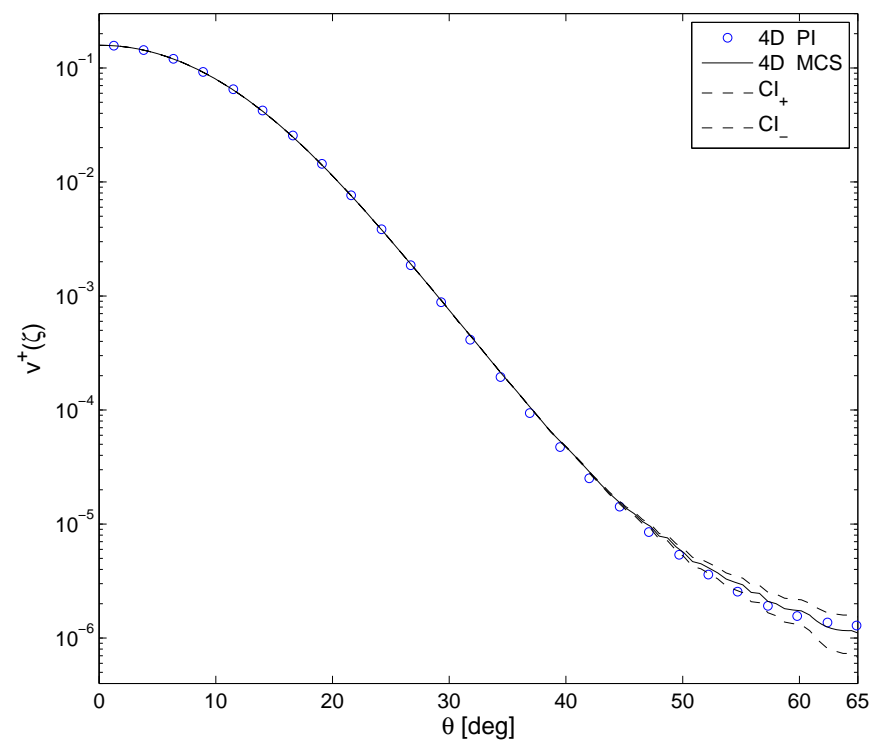

Figure 6: Upcrossing rates obtained by the 4D PI method and MCS.

\section{CONCLUSIONS}

In this work, a 4D Markov dynamic system is generated in order to describe the rolling behavior of a vessel subjected to random wave excitation. Based on the Markov property of the dynamic system, a 4D PI scheme is introduced to solve the high-dimensional FP equation. Comparisons of the response statistics obtained by the 4D PI method and MCS demonstrate the numerical robustness and accuracy of the 4D PI in calculating the statistics of the high roll response levels when subjected to random wave excitation. 


\section{REFERENCES}

[1] J. Roberts, M. Vasta, Markov modelling and stochastic identification for nonlinear ship rolling in random waves. Philosophical Transactions of the Royal Society of London. Series A: Mathematical, Physical and Engineering Sciences, 358, 1917-1941, 2000.

[2] W. Chai, J. Fan, R. Zhu, X. Huang, G. Miao, Calculation of Ship Rolling Capsizing Probability by Path Integral Solution. Journal of Shanghai Jiaotong University, 28, 317322, 2013.

[3] L. Dostal, E. Kreuzer, Probabilistic approach to large amplitude ship rolling in random seas. Proceedings of the Institution of Mechanical Engineers, Part C: Journal of Mechanical Engineering Science, 225, 2464-2476, 2011.

[4] Z. Su, J.M. Falzarano, Markov and Melnikov based methods for vessel capsizing criteria. Ocean Engineering, 64, 146-152, 2013.

[5] Jr, B. Spencer, L. Bergman, On the numerical solution of the Fokker-Planck equation for nonlinear stochastic systems. Nonlinear Dynamics, 4, 357-372, 1993.

[6] S.F. Wojtkiewicz, E.A. Johnson, L.A. Bergman, M. Grigoriu, Jr, B.F. Spencer, Response of stochastic dynamical systems driven by additive Gaussian and Poisson white noise: Solution of a forward generalized Kolmogorov equation by a spectral finite difference method. Computer methods in applied mechanics and engineering, 168, 73-89, 1999.

[7] W. Chai, A. Naess, B.J. Leira, Filter models for prediction of stochastic ship roll response. Probabilistic Engineering Mechanics, 41, 104-114, 2015.

[8] E. Mo, Nonlinear stochastic dynamics and chaos by numerical path integration. Norwegian University of Science and Technology, PhD Thesis, 2008.

[9] A. Naess, J. Johnsen, Response statistics of nonlinear, compliant offshore structures by the path integral solution method. Probabilistic Engineering Mechanics, 8, 91-106, 1993.

[10] W. Chai, A. Naess, B.J. Leira, Stochastic Dynamic Analysis and Reliability of a Vessel Rolling in Random Beam Seas. Journal of Ship Research, 59, 113-131, 2015.

[11] Z. Su, Nonlinear response and stability analysis of vessel rolling motion in random waves using stochastic dynamical systems. Texas A\&M University, PhD Thesis, 2012.

[12] W. Chai, A. Naess, B.J. Leira, Stochastic nonlinear ship rolling in random beam seas by the path integration method. Probabilistic Engineering Mechanics, in press, 2015. 